\title{
Graphene sandwiches as a platform for broadband molecular spectroscopy
}

\author{
Yan Francescato, ${ }^{*, \dagger} \ddagger$ Vincenzo Giannini, ${ }^{\dagger}$ Jingjing Yang, ${ }^{\ddagger}$ Ming Huang, ${ }^{\ddagger}$ and \\ Stefan A. Maier
}

The Blackett Laboratory, Imperial College London, London SW7 2AZ, United Kingdom, and Wireless Innovation Lab of Yunnan University, Kunming 650091, PR China

E-mail: yan.francescato10@imperial.ac.uk

\begin{abstract}
Sensing is to date one of the most successful applications of surface plasmons thanks to the exceptional field amplification and sensitivity of these modes in metallic nanostructures. Here we introduce a promising detection scheme based on the propagation of strongly confined anti-bonding plasmons supported by graphene sandwiches. Instead of measuring changes in the refractive index or enhancing a restricted number of molecular absorption lines, the proposed device can recover an extended portion of the infrared spectrum of a molecule. Moreover, the extreme compression of light in graphene means that a diluted $2 \mathrm{~nm}$-thick analyte can cause up to $3 \mathrm{~dB}$ intensity changes. The broadband capability and sensitivity also imply that one can easily identify different chemicals in a mixture and extract their respective concentration. We conclude by presenting a simple experimental set-up based on this mechanism for infrared spectroscopy which could become a cheap Fourier transform infrared accessory and an alternative to crystal-based attenuated total reflection spectroscopy.
\end{abstract}

\footnotetext{
* To whom correspondence should be addressed

${ }^{\dagger}$ The Blackett Laboratory, Imperial College London, London SW7 2AZ, United Kingdom

${ }^{\ddagger}$ Wireless Innovation Lab of Yunnan University, Kunming 650091, PR China
} 
Keywords: Broadband sensing, infrared spectroscopy, graphene plasmons, plasmonic sensing.

Sensing has always been an important driving force and is still subjected to an intense research in the nanophotonic community. This stems in large part from the understanding of the role of localized plasmons in the gigantic signal amplification observed in surfaceenhanced spectroscopy (SES) ${ }^{1}$ such as SERS $^{2}$ (for Raman scattering) and SEIRA $^{3}$ (for infrared absorption). Subsequent commercial applications in the mid-nineties of pregnancy tests based on metal colloids ${ }^{4}$ finished to establish plasmonics has a cheap and powerful detection technique. The interest in surface plasmons, these collective oscillations of the conduction electrons in conductors, lies in the intense hot spots that can be found in their vicinity as well as the sensitivity of these modes to their surrounding environment. This led on one hand to SES where molecules near metallic nanostructures exhibit increased vibrational fingerprints. ${ }^{5-8}$ On another hand, the plasmonic modes in chemically prepared metallic colloids, ${ }^{9-11}$ or more advanced and flexible geometries obtained by top-down fabrication techniques, ${ }^{12,13}$ will experience drastic spectral shifts upon molecular adsorption. Both methods have proved robust and commercially viable, however they suffer from some limitations. For example in SEIRA, the resonances in metal nanoparticles are much narrower than the whole spectrum of molecules and so only a few vibrational modes will be amplified. ${ }^{6-8,14,15}$ In index sensing, the whole specificity is provided by the chemical preparation of the metal surface, which can be long and tedious, and anything which can bind will produce a signal.

In order to circumvent both issues, we propose here to use the broadband field amplification provided by propagating surface plasmons in the infrared. This gives access to an extended part of a molecular spectrum allowing its clear identification in a read out fashion. To further improve the sensitivity we additionally rely on plasmonic excitations in doped graphene.

Graphene has emerged as an exciting platform for condensed matter physics, ${ }^{16}$ and its optical $^{17,18}$ and electrical ${ }^{19}$ properties are fascinating, as it exhibits for instance room tem- 
perature quantum Hall effect and quantized transparency. ${ }^{20,21}$ As much as pristine graphene which is attractive because of its Dirac cone dispersion and gaplessness, ${ }^{22}$ doped graphene allows a strict control over the interband and intraband transitions which can take place. ${ }^{23,24}$ This tunability is foreseen as one of the key advantage of graphene over metals for applications in nanophotonics. ${ }^{25}$ However, there is more to graphene plasmonics than the appealing operational range covering the near-infrared to the THz regime. ${ }^{26-28}$ The most impressive of which is probably the extreme compression of these surface waves with lateral confinement extending only a few nanometres away from the graphene sheet. ${ }^{29-33}$ Furthermore, the transparency of graphene gives rise to particular hybridized modes in coupled sheets. ${ }^{34}$ This is even more striking in paired ribbons where the contribution from the edges can result in unexpected field profiles. ${ }^{35,36}$ The unmatched localization of light in graphene nanostructures also implies a much improved sensitivity to the direct environment than for metal-based plasmonics.

\section{Principle of broadband sensing}

In our previous work, we investigated thoroughly the strongly confined plasmon modes which are supported by graphene ribbon sandwiches. ${ }^{36}$ In particular, we showed that for small enough gaps, such geometry can sustain an anti-bonding waveguide mode in which the localized field lies within $2 \mathrm{~nm}$ from the external surface, see Figure 1a for an example. Here we prove that this mode is well adapted for a promising sensing scheme based on propagating surface plasmons polaritons (SPPs). Unlike existing plasmonic detection techniques which rely on spectral shifts caused by changes in the environment refractive index, ${ }^{37,38}$ the proposed method rely on the broadband localization capability of propagating SPPs. The basic principle is to measure the intensity of the SPPs after propagation through a thin layer of the analyte compared with that of a clear channel. Since molecules exhibit strong absorption lines in the infrared, the propagation losses will be greater at those frequencies. Therefore the spectral intensity profile of the SPPs at the exit of the analyte will directly map its 
absorption spectrum. This reminds of the more classical way of measuring the absorbance of samples. One would pass light through a given thickness of the material and extract from the reduced intensity at the output the amount of absorption light suffered. The main difference lies in the volume of analyte required. Indeed, since SPPs are compressed both in the lateral and propagation directions, the volume of the analyte for a similar signal to that obtained with free space light is drastically reduced. Alternatively, one can realise that the infrared absorption of a molecule is proportional to the intensity of the field. One of the main appeal of SPPs being the gigantic local field enhancement, the absorption cross-section of an analyte bathed by SPPs is increased by a few orders of magnitude. While typical SEIRA is also based on the field enhancement provided by metallic nanostructures, the associated localized plasmons in those are relatively narrowband spectrally speaking. As a result, only a few absorption peaks mostly located on the redside of the plasmonic resonance are generally observed. On the reverse, SPPs are intrinsically broadband, existing at all frequencies below their electrostatic asymptote at $\omega_{s p p}=\omega_{p} / \sqrt{2}$ where $\omega_{p}$ is the plasma frequency (or at $\omega_{\text {spp }}=1.667 \mu / \hbar$ for doped graphene where $\mu=E_{F}$ is the chemical potential or Fermi level and $\hbar$ Planck's constant). Therefore one can benefit from their enhancement capability over a large portion of the electromagnetic spectrum as long as they can be excited. This last step can prove difficult to achieve as SPPs usually exhibit a strong dispersion with energy. In the situation where the frequency window of interest is at sufficiently small energies compared to $\omega_{\text {spp }}$ and not too close to the cut-off frequency in the case of guided modes, the dispersion is sufficiently flat to allow an efficient coupling over a broad range. Last, let us stress that although the proposed sensing scheme could be performed with SPPs on metal surfaces alike, the large compression of SPPs in graphene allows to probe a much smaller volume of analyte. With the presented geometry, a $10 \%$ concentrated $2 \mathrm{~nm}$-thin analyte layer leads to a $3 \mathrm{~dB}$ signal after only a $10 \mu \mathrm{m}-$ long SPP propagation. 


\section{Results and discussion}

\section{Guided plasmon modes in graphene sandwiches}

The doping of graphene induces the presence of free carriers which can be stimulated collectively to form surface plasmon polaritons similarly to those supported by metals. Those excitations propagate along the plane of the graphene sheet and are confined in the perpendicular direction hence their appellation as surface waves. ${ }^{39}$ When the sheet is patterned into ribbons, the finite width leads to an additional confinement in the lateral direction giving rise to guided modes such as the ones found in dielectric waveguides. As the order of the waveguide mode increases, the ratio between the lateral and longitudinal components of the wavevector increases until it reaches the condition equivalent to that of total reflection at which point the modes cease to be bounded. Thanks to its vanishing thickness and linear dispersion, ribbons made out of graphene exhibit a particular mode spectrum which was investigated in detail by Nikitin and co-workers. ${ }^{29}$ Although sheets which are vertically spaced produce the expected symmetric and anti-symmetric combinations of the surface plasmons ${ }^{34}$ known as short and long-range modes within the plasmonic community, ${ }^{40}$ the transparency of graphene and the extremely confined modes propagating along the edges ${ }^{41,42}$ complicate and enrich markedly the hybridization process. Christensen et al. studied thoroughly those modes focusing on narrow ribbons where the contribution from the edges was greatest and derived a useful scaling law in order to predict their spectral behaviour in paired ribbons. ${ }^{35}$ We further considered wide graphene ribbon sandwiches where we showed that waveguided modes were forming bonding and antibonding supermodes along with hybridized edge modes. ${ }^{36}$ While the bonding waveguide modes in which the field is concentrated within the gap region were proposed as appealing building blocks for nanocircuitry, the antibonding waveguide modes were seen as a potential plateform for sensing thanks to the field confinement taking place right at the surface of the sandwich. We will now prove that the latter is indeed attractive for molecular detection and moreover particularly well adapted for the 
proposed broadband sensing scheme.

Drawing on our previous results, we consider doped graphene with a chemical potential $\mu=E_{F}=0.5 \mathrm{eV}$ and a charge carrier scattering rate $\Gamma=0.1 \mathrm{meV}$. The two graphene ribbons are separated by a $2 \mathrm{~nm}$-thick dielectric spacer with $n=1.5$ and toped with a $2 \mathrm{~nm}$ thick analyte layer, see Figure 1b. Furthermore, following the designing guidelines provided in Reference, ${ }^{36}$ the ribbons width is set to $600 \mathrm{~nm}$ in order for the sandwich to support anti-bonding waveguide modes, the field profile of which is shown in Figure 1a. The top

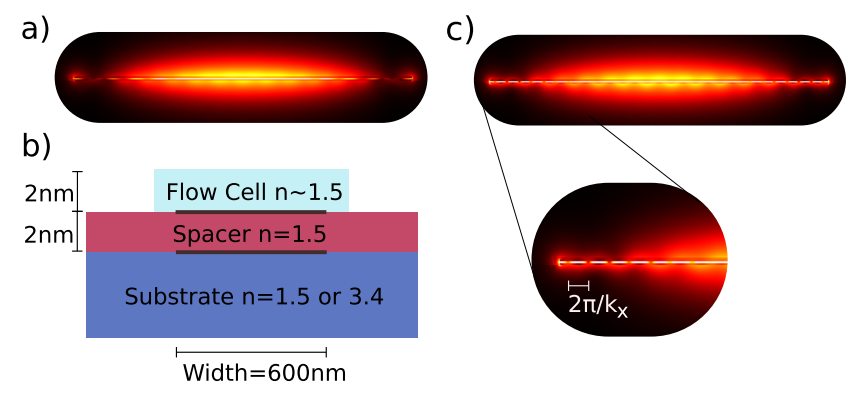

Figure 1: a) Field distribution of the first order antibonding waveguided plasmon mode in the proposed graphene sandwich at $\nu=33 \mathrm{THz}$ with $n_{\text {sub }}=3.4$. b) Cross-section of the geometry investigated consisting of two vertically spaced graphene ribbons $600 \mathrm{~nm}$ wide on a $n=1.5$ or 3.4 index substrate and covered by a $2 \mathrm{~nm}$-thin analyte layer with index close to 1.5, the spacer is also $2 \mathrm{~nm}$ thin with $n=1.5$. c) Field distribution of the same mode than a) at $\nu=32.4 \mathrm{THz}$ where phase matching leads to the stimulation of a high-order bonding waveguided plasmon mode with lateral wavevector $k_{x}$.

layer has an index of 1.5 for the reference channel calculation and a $10 \%$ diluted complex index $\tilde{n} \sim 1.5$ extracted from the absorption spectrum of phenol in the case of the analyte, see the Methods section for full details. We focus our investigation here to the frequency range $31-40 \mathrm{THz}\left(1033-1333 \mathrm{~cm}^{-1}\right.$ or $\left.9.7-7.5 \mu \mathrm{m}\right)$ corresponding to a region of the infrared where most molecules exhibit fingerprint-like absorption peaks. The guided plasmon modes are calculated by finite-element method (FEM), see the Methods section, and are defined on one side by their propagation constant (or wavevector) $\beta$ which defines the amount of compression of the light and on the other side by their propagation length $L$ which quantifies their propagation loss. The plasmon modes have a wavelength $\lambda_{p}=\lambda_{0} / \beta$ and an evanescent decay away from the graphene $\delta \sim 1 / 2 \beta$. Their propagation length is defined as a $1 / \mathrm{e} \sim 37 \%$ 
decrease in intensity.

Figure 2 presents the normalized wavevectors (to the incident light wavevector $k_{0}$, full lines) and propagation constants (to the plasmon wavelengths $\lambda_{p}$, dashed lines) of the first order anti-bonding waveguided plasmon modes in the case of a $n=1.5$ (in blue) and 3.4 (red line) substrate without the analyte. The propagation length is also shown in absolute values in Figure 4. Apart from the very distinct dispersions of the two substrates cases, which

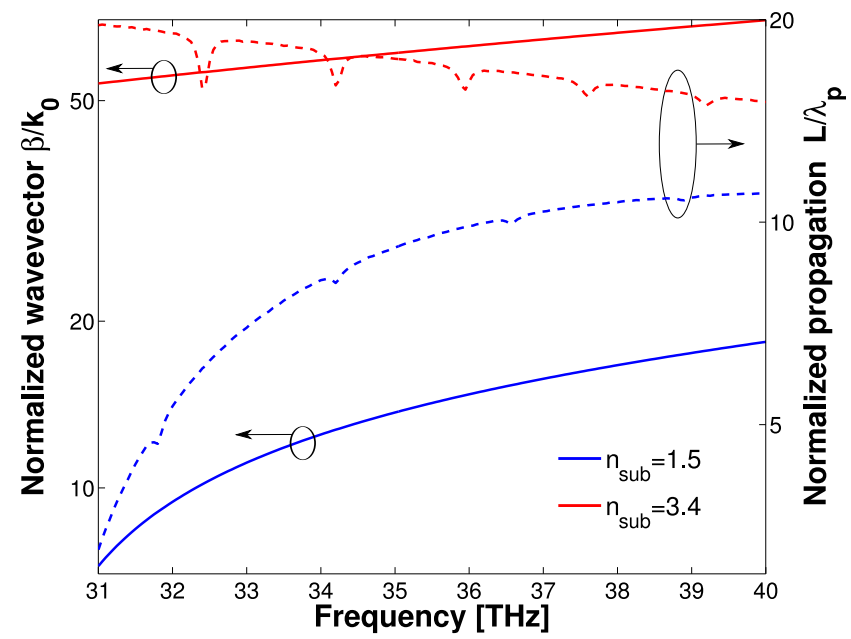

Figure 2: Normalized propagation constant $\beta / k_{0}$ (full lines) and normalized propagation length $L / \lambda_{p}$ (dashed lines) of the first order antibonding mode in the geometry presented in Figure $1 \mathrm{~b}$. The blue line is for $n_{\text {sub }}=1.5$ and the red curve is for $n_{\text {sub }}=3.4$.

is caused by a shift of the cut-off frequency of the mode due to the substrate index, let us highlight the existence of peculiar dips which appear in the propagation length curves even in the absence of an absorber. As we will see in the following, these modes bear no influence on the functioning of the device and are normalized out in the final results. They are caused by phase matching between the anti-bonding wageguide mode and high orders bonding modes which are propagating within the gap, as shown in Figure 1c. Those are strictly confined to the gap region and in consequence do not sense the molecule. This is why their effect cancels out when normalizing by the reference channel. These dips are regularly spaced at frequencies where the propagation constant of one of the bonding modes match the one of the antibonding plasmon. ${ }^{36}$ 


\section{Robustness of the modes}

To further investigate the feasibility of such scheme, we have considered the case of a low and high index substrates as illustrated in the previous figure. The results prove that a symmetric environment is not necessary for the modes to exist, more, a substrate with a higher index leads to a reduced plasmon wavelength and therefore an increased sensitivity of the device, as we will see. With the parameters chosen here, it can be concluded that the high index substrate is also more appropriate because it leads to a smaller dispersion improving the coupling efficiency from free-space light.

Next, we analyse variations in the index of the top layer and background index in the range 1-2 and conclude that the modes are extremely robust. Figure 3 reports the normalized propagation constant (full lines) and propagation length (now normalized to the free space wavelength $\lambda_{0}$, dashed lines) for the substrate with $n=3.4$ and let the 2 nm-thick spacer index fixed at $n=1.5$. For the sake of clarity we show here the variations only at the first $(31 \mathrm{THz}$, in blue) and last frequency $(40 \mathrm{THz}$, in red) investigated. We span on one hand the index of the 2 nm-thick top layer keeping the background to $n=1$, see Figure $3 \mathrm{a}$, and on the other hand the index of the background with $n_{\text {layer }}=n_{\text {mol }}=1.5$, see Figure 3b. While we change the index of the whole background, due to the small extent of the plasmon field away from the graphene, this is similar to a change of the flow cell index. There are two main conclusions from Figure 3. First of all, the effect of both index changes on the modes is very moderate and purely monotonic. This implies that the modes are extremely robust to inhomogeneities and to any asymmetry in the indices of the spacer and top layers. More importantly, since the effect is merely a flat renormalization of the propagation characteristics, the reference channel indices do not need to be identical to those of the sample channel as long as their frequency dispersion is similar. 

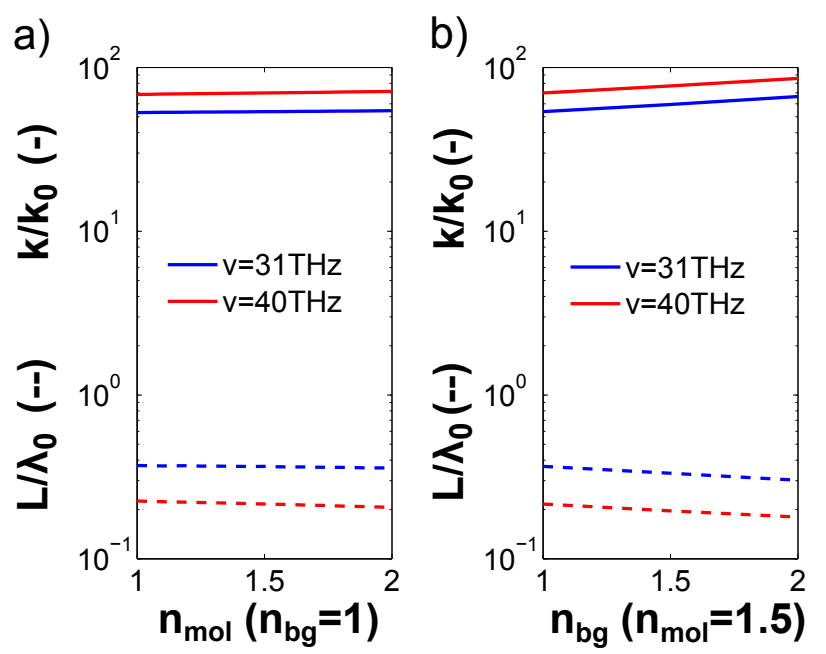

Figure 3: Normalized propagation constants (full lines) and propagation lengths (dashed lines) of the antibonding waveguided plasmon mode depending on the index of the surrounding at 31 (blue) and $40 \mathrm{THz}$ (red). a) Effect of the index of the $2 \mathrm{~nm}$-thin analyte layer with a fixed index background $n_{b g}=1$. b) Effect of the index of the background (or flow cell, as discussed in the running text) while keeping $n_{m o l}=1.5$.

\section{Effect of the analyte on the propagation}

We have shown that the device can work properly even in the case where the reference and sample channels indices were to be different and that it was rather robust to inhomogeneities. We now consider the effect of the analyte on the propagation of the antibonding waveguide mode in order to assess its performance. Details of the model used to describe the analyte layer are given in the Methods section. Figure 4 shows the propagation distance in absolute values after which the plasmons intensity is 1 /e for the low ( $n=1.5$, in blue) and high ( $n=3.4$, in red) index substrates with (dashed lines) and without (full lines) the analyte layer. As one can see, due to an increased compression of the mode due to the higher index, the plasmons supported by the $n=3.4$ substrate propagate less. However, since there is respectively more optical cycles per unit length, these modes are more sensitive as is apparent in Figure 5. Note again the dips caused by the phase matching between the first anti-bonding waveguide mode and high order bonding waveguide modes. We observe that the effect of the molecule is negligible on the propagation constants (not shown) as can be expected from its very small variation of refractive index. However, this is not the case for the 
propagation losses which are drastically different in presence of the molecule. Moreover, one can already guess that the difference between the reference and sample channels is directly related to the absorption spectrum of the molecule. The intensity of the surface plasmon

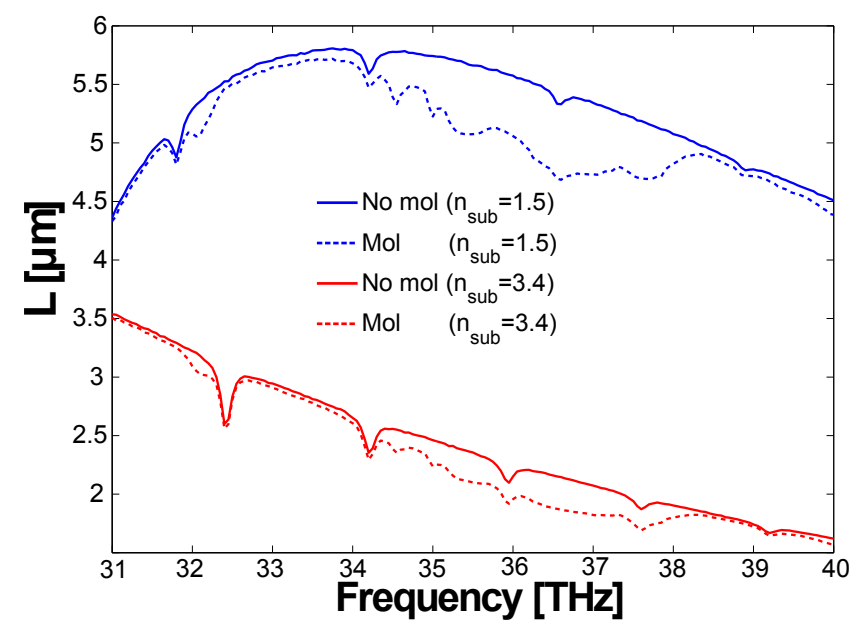

Figure 4: Propagation length in absolute values of the same modes presented in Figure 2. Blue lines are for $n_{\text {sub }}=1.5$ and red curves are for $n_{\text {sub }}=3.4$. Full lines are for a molecule-free reference channel and dashed curves for the case of the sample channel.

along the propagation direction is $I=I_{0} \exp (-x / L)$ where $x$ is the distance. Therefore at a fixed position one is able to compare the intensity of the plasmon through a reference channel and through the analyte. The obtained normalized signal $I_{m o l} / I_{r e f}$ is presented in Figure 5 for both substrate cases ( $n=1.5$ in full lines and $n=3.4$ in dashed lines) at three different position 1 (green), 5 (red) and $10 \mu \mathrm{m}$ (blue) from the input. The left axis is given in $\mathrm{dB}=10 \times \log \left(I_{m o l} / I_{r e f}\right)$. Let us remind here that the considered analyte consists in a $2 \mathrm{~nm}$-thin $10 \%$ diluted molecular species. Nevertheless, a $10 \mu \mathrm{m}$-long sample can produce a gigantic $3 \mathrm{~dB}(50 \%)$ drop in intensity with the proposed geometry. Due to this exceptional sensitivity we predict that even partially formed monolayers could be easily detected with a device based on this principle. Furthermore, the broadband signal corresponds exactly to the infrared absorption spectrum of the molecule, shown in the inset of Figure 5, and allows in consequence a clear chemical analysis. In the case of a mixture, thanks to the extended frequency range one can access, it is also possible to identify the different chemicals present and extract their respective concentrations. 


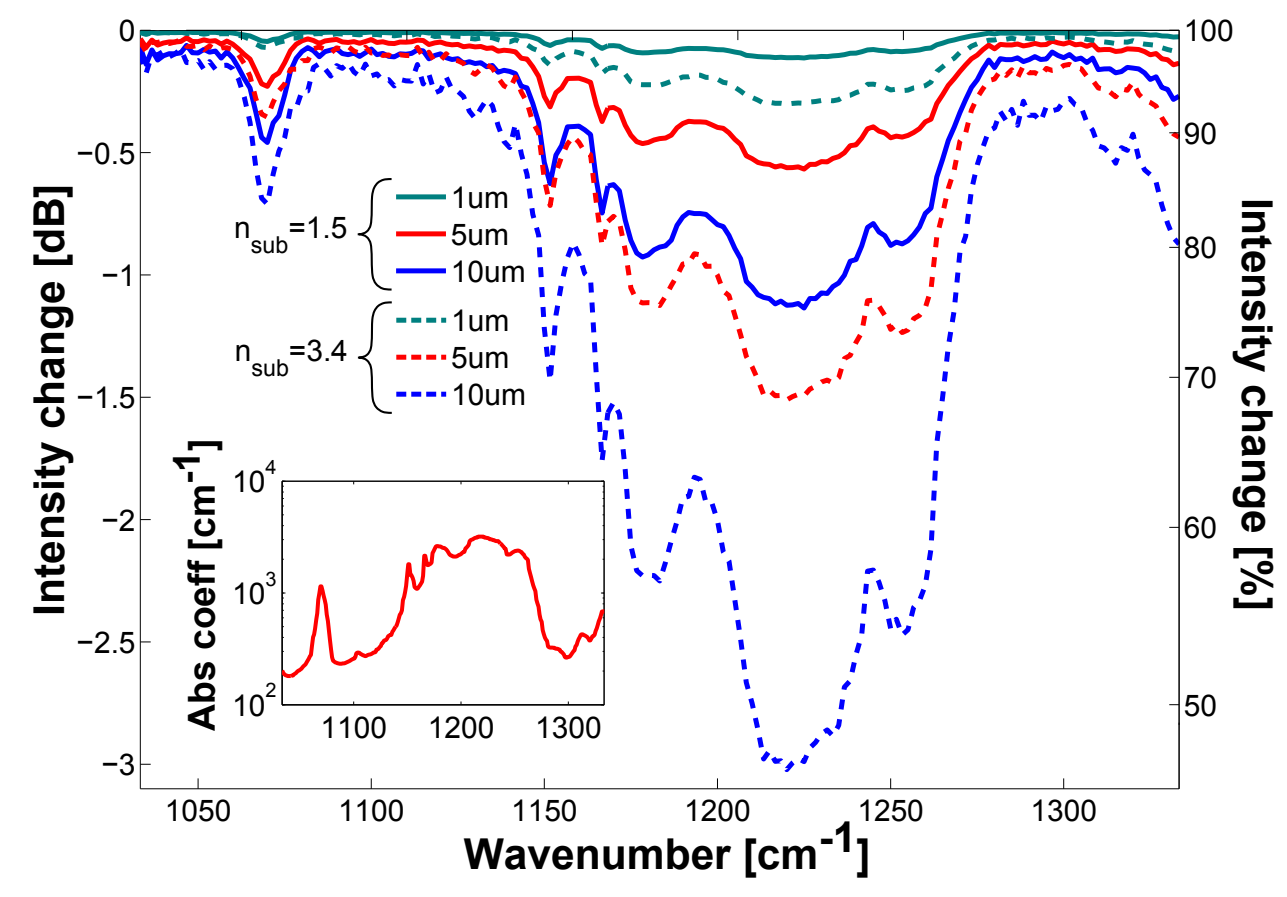

Figure 5: Intensity changes caused by the presence of the analyte in $\mathrm{dB}\left(10 \times \log \left(I_{\text {mol }} / I_{\text {ref }}\right)\right.$, left axis) and percent $\left(100 \times I_{m o l} / I_{r e f}\right.$, right axis) for the low (full lines) and high index (dashed lines) substrate case. The measured intensity drop is for an input-output distance of 1 (green), 5 (red) and $10 \mu \mathrm{m}$ (blue). The inset shows the absorption coefficient in log scale of the analyte in the same frequency region as the main axis. 


\section{Potential implementation as an FTIR accessory}

Based on these promising results we propose next a device making use of the broadband principle we just introduced and the extraordinary sensitivity of anti-bonding waveguide modes in graphene sandwiches. The most simple experimental set-up would rely on a FourierTransform InfraRed (FTIR) spectrometer which is a very common tool nowadays to perform chemical analysis in the infrared. This apparatus would provide for the broadband source and the detection means. The device would then simply be an accessory to place under the microscope and would consist of one or two built-in channels, see Figure 6 . In the former case a reference signal should first be acquired. Similarly to the geometry shown in Figure 1b, the substrate would be covered by a graphene sheet and patterned by lithography into a ribbon. The width of the latter should be tuned to support guided plasmon modes within the desired frequency range according to the doping of the graphene following the guidelines of Reference. ${ }^{36}$ It would be followed by the deposition of a spacer for instance by spin-coating and a second patterned ribbon. The whole could then by covered with a flow cell in order to be reusable. A reference solution would first be circulated followed by that of the solution to be tested. Thanks to the relatively small wavevectors (compared to usual SPPs in graphene) of the modes considered here and their rather moderate dispersion away from their respective cut-off frequency, their excitation from free-space light can easily be achieved through grating coupling. ${ }^{43,44}$ For the lowest index substrate $(n=1.5)$, the grating period should be of the order of $\Lambda=\lambda_{0} k_{0} / \beta \sim 10 \mu \mathrm{m} / 20=500 \mathrm{~nm}$ and not smaller than $100 \mathrm{~nm}$ in any case. The grating allows to couple light to the confined surface waves thanks to the extra momentum it provides. At the output, a second grating is placed which scatters the plasmons back to freespace and are collected by the detector. For increased coupling, one could also make use of trapezoidal gratings in which the varying width broadens considerably the optical response ${ }^{45}$ or cascaded gratings. ${ }^{46}$ Alternatively, the phase-matching at the input and output can be provided by small scatters such as metallic nanoparticles with sizes $a \sim 1 / \beta$ which are easily achieved through standard lithography. We foresee that a device based on this broadband 
a) Top View

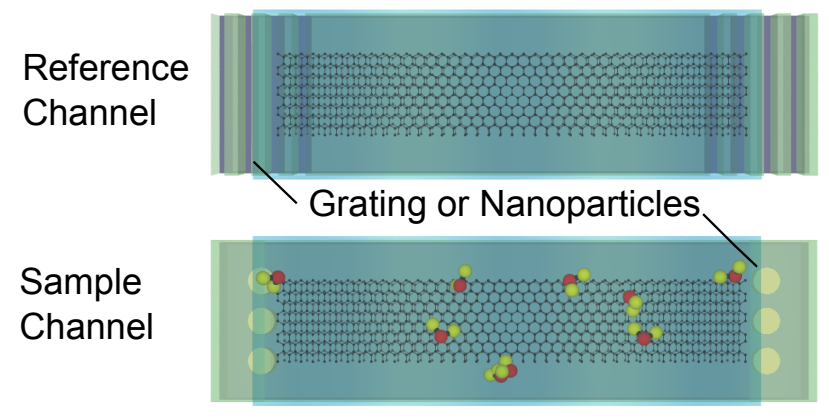

b) Side View
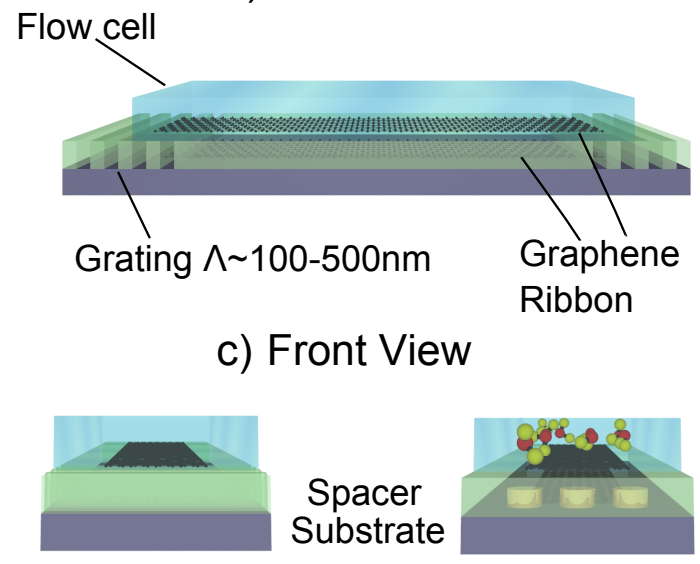

Figure 6: Design of a device based on the ultra sensitive broadband capability of the proposed graphene sandwich structure. It consists in a reference and single channel where a flow cell is placed on top of the sandwich in order to circulate liquid samples. The coupling of the free-space light to the plasmon modes is enabled by gratings with period which can be as large as $500 \mathrm{~nm}$ for a low index substrate or via the broad plasmonic response of metallic nanoparticles. 
sensing capability could serve as cheap alternative to Attenuated Total Reflection (ATR) schemes which rely on expensive high-quality semiconducting crystals.

\section{Conclusion}

In conclusion, we have demonstrated a promising method to measure the infrared absorption of chemicals based on propagating surface plasmon modes. Above all, it differs from classical plasmonic sensing because of its broadband character which allows to extract an extended portion of a molecular absorption spectrum. This is in strong contrast with index sensing, where one measure the induced spectral shift of a plasmonic or cavity mode due to chemical adsorption, or SEIRA in which only a few vibrational lines of a molecule are amplified. Although the proposed broadband sensing scheme can make use of any confined surface modes, we further show that particular waveguided modes in graphene sandwiches can lead to an unprecedented sensitivity. Indeed our calculations suggest that the anti-bonding waveguide mode in a $2 \mathrm{~nm}$-spaced sandwich of two graphene ribbons exhibit an extremely intense field localization directly at its external face. By covering this structure with a 2 nm-thick diluted molecular species, the infrared absorption spectrum of the chemical can be recovered with up to $3 \mathrm{~dB}$ signal. This exceptional sensitivity coupled with the broadband capability also means that one can identify all the chemical species in presence and their respective concentration in a mixture. These encouraging results lead us to propose a simple device based on this sensing principle which could be conceived as an accessory for FTIR microscopes and an alternative to ATR-crystal spectroscopy.

\section{Methods}

The simulation results presented here were obtained from the FEM package COMSOL Multiphysics. The model considers an infinitely long structure in the propagation direction and calculate the waveguided modes that are supported. Graphene is approximated by an effec- 
tive permittivity $\varepsilon=1+\mathrm{i} \sigma / \varepsilon_{0} \omega t$ where $\varepsilon_{0}$ is the permittivity of vacuum, $\omega=2 \pi \mathrm{c} / \lambda_{0}$ the angular frequency of light and $t=1 \mathrm{~nm}$ the selected thickness of the graphene ribbons. ${ }^{47}$ $\sigma$ is the surface conductivity of graphene as obtained from the local random phase approximation of the Kubo formula ${ }^{47}$ at room temperature and with a Fermi level $E_{F}=0.5 \mathrm{eV}$ and charge carrier scattering rate $\Gamma=0.1 \mathrm{meV}$. The triangular mesh was set to $t / 2$ in the volume surrounding the nanostructure which together with the graphene effective thickness was sufficient for fully converged solutions as attested by convergence tests at smaller mesh sizes and thicknesses and as can be expected for such a deeply sub-wavelength scale.

The $2 \mathrm{~nm}$-thick top layer is chosen to be either purely dielectric with $n=1.5$ in which case it is considered sample-free or with a complex index $\tilde{n}$ which is given by the molecule absorption. We choose here a phenol molecule, the transmittance spectrum of which we obtained from the National Institute of Standards and Technology (NIST) (http://webbook.nist. gov/cgi/cbook . cgi?ID=C108952\&Units=SI\&Type=IR-SPEC\&Index=1\#IR-SPEC). From BeerLambert's relation $T=\exp (-\alpha x)$, where $x$ is the path length of light within the solution, the absorption coefficient $\alpha$ can be extracted, and is plotted as inset in Figure 5. KramersKronig's relations allow then to calculate the corresponding refractive index thanks to causality requirements. The complex index of the molecule finally reads

$$
\tilde{n}(\omega)=1.5+\frac{\mathrm{c}}{\pi} \mathcal{P} \int_{0}^{\infty} \frac{\alpha\left(\omega^{\prime}\right)}{\omega^{\prime 2}-\omega^{2}} \mathrm{~d} \omega^{\prime}+\mathrm{i} \frac{\alpha(\omega) \mathrm{c}}{2 \omega}
$$

where $\mathcal{P}$ indicates the principal part of the undefined integral and $\mathrm{c}$ is the speed of light in vacuum. The layer defined in this fashion is a good approximation of a monolayer with a phenol-like vibrational spectrum. To stress the sensitivity of our sensor we only use a phenol concentrated to $10 \%$ in the presented results, i.e. $n_{m o l}=1.5+0.1 \tilde{n}$. 


\section{Acknowledgement}

Y.F., V.G. and S.M. acknowledge support from the UK Engineering and Physical Sciences

Research Council (EPSRC). J.Y. and M.H. would like to thank the National Natural Science Foundation of China (Grant Nos. 61161007, 61261002) and China Postdoctoral Science Foundation (Grant No. 2013M531989).

\section{References}

(1) Moskovits, M. Surface-enhanced spectroscopy. Rev. Mod. Phys. 1985, 57, 783-826.

(2) Moskovits, M. Surface-enhanced Raman spectroscopy: a brief retrospective. J. Raman Spectrosc. 2005, 36, 485-496.

(3) Osawa, M. Near-field optics and surface plasmon polaritons; Topics in applied physics; Springer-Verlag: Berlin, 2001; Vol. 81; pp 163-187.

(4) Stockman, M. I. Nanoplasmonics: The physics behind the applications. Phys. Today 2011, 64, 39-44.

(5) Kneipp, K.; Wang, Y.; Kneipp, H.; Perelman, L.; Itzkan, I.; Dasari, R.; Feld, M. Single molecule detection using surface-enhanced Raman scattering (SERS). Phys. Rev. Lett. 1997, 78, 1667-1670.

(6) Neubrech, F.; Pucci, A.; Cornelius, T. W.; Karim, S.; Garcia-Etxarri, A.; Aizpurua, J. Resonant Plasmonic and Vibrational Coupling in a Tailored Nanoantenna for Infrared Detection. Phys. Rev. Lett. 2008, 101.

(7) Adato, R.; Yanik, A. A.; Amsden, J. J.; Kaplan, D. L.; Omenetto, F. G.; Hong, M. K.; Erramilli, S.; Altug, H. Ultra-sensitive vibrational spectroscopy of protein monolayers with plasmonic nanoantenna arrays. Proc. Natl. Acad. Sci. U.S.A. 2009, 106, 1922719232. 
(8) Pryce, I. M.; Aydin, K.; Kelaita, Y. A.; Briggs, R. M.; Atwater, H. A. Highly Strained Compliant Optical Metamaterials with Large Frequency Tunability. Nano Lett. 2010, $10,4222-4227$.

(9) Homola, J.; Yee, S.; Gauglitz, G. Surface plasmon resonance sensors: review. Sens. Actuators, B 1999, 54, 3-15.

(10) Willets, K. A.; Van Duyne, R. P. Localized surface plasmon resonance spectroscopy and sensing. Annu. Rev. Phys. Chem. 2007, 58, 267-297.

(11) Anker, J. N.; Hall, W. P.; Lyandres, O.; Shah, N. C.; Zhao, J.; Van Duyne, R. P. Biosensing with plasmonic nanosensors. Nat. Mater. 2008, 7, 442-453.

(12) Aćimović, S. S.; Kreuzer, M. P.; González, M. U.; Quidant, R. Plasmon Near-Field Coupling in Metal Dimers as a Step toward Single-Molecule Sensing. ACS Nano 2009, 3, 1231-1237.

(13) Cetin, A. E.; Altug, H. Fano Resonant Ring/Disk Plasmonic Nanocavities on Conducting Substrates for Advanced Biosensing. ACS Nano 2012, 6, 9989-9995.

(14) Aouani, H.; Sipova, H.; Rahmani, M.; Navarro-Cia, M.; Hegnerova, K.; Homola, J.; Hong, M.; Maier, S. A. Ultrasensitive Broadband Probing of Molecular Vibrational Modes with Multifrequency Optical Antennas. ACS Nano 2013, 7, 669-675.

(15) Limaj, O.; D’Apuzzo, F.; Di Gaspare, A.; Giliberti, V.; Domenici, F.; Sennato, S.; Bordi, F.; Lupi, S.; Ortolani, M. Mid-Infrared Surface Plasmon Polariton Sensors Resonant with the Vibrational Modes of Phospholipid Layers. J. Phys. Chem. C 2013, $117,19119-19126$.

(16) Castro Neto, A. H.; Guinea, F.; Peres, N. M. R.; Novoselov, K. S.; Geim, A. K. The electronic properties of graphene. Rev. Mod. Phys. 2009, 81, 109-162. 
(17) Gusynin, V. P.; Sharapov, S. G.; Carbotte, J. P. Magneto-optical conductivity in graphene. J. Phys.: Condens. Matter 2007, 19, 026222.

(18) Bonaccorso, F.; Sun, Z.; Hasan, T.; Ferrari, A. C. Graphene photonics and optoelectronics. Nat. Photonics 2010, 4, 611-622.

(19) Avouris, P. Graphene: Electronic and Photonic Properties and Devices. Nano Lett. 2010, 10, 4285-4294.

(20) Gusynin, V. P.; Sharapov, S. G. Transport of Dirac quasiparticles in graphene: Hall and optical conductivities. Phys. Rev. B 2006, 73, 245411.

(21) Nair, R. R.; Blake, P.; Grigorenko, A. N.; Novoselov, K. S.; Booth, T. J.; Stauber, T.; Peres, N. M. R.; Geim, A. K. Fine Structure Constant Defines Visual Transparency of Graphene. Science 2008, 320, 1308.

(22) Geim, A. K. Graphene: Status and Prospects. Science 2009, 324, 1530-1534.

(23) Wang, F.; Zhang, Y.; Tian, C.; Girit, C.; Zettl, A.; Crommie, M.; Shen, Y. R. Gatevariable optical transitions in graphene. Science 2008, 320, 206-209.

(24) Chen, C.-F.; Park, C.-H.; Boudouris, B. W.; Horng, J.; Geng, B.; Girit, C.; Zettl, A.; Crommie, M. F.; Segalman, R. A.; Louie, S. G.; Wang, F. Controlling inelastic light scattering quantum pathways in graphene. Nature 2011, 471, 617-620.

(25) Bao, Q.; Loh, K. P. Graphene Photonics, Plasmonics, and Broadband Optoelectronic Devices. ACS Nano 2012, 6, 3677-3694.

(26) Koppens, F. H. L.; Chang, D. E.; García de Abajo, F. J. Graphene Plasmonics: A Platform for Strong Light-Matter Interactions. Nano Lett. 2011, 11, 3370-3377.

(27) Grigorenko, A. N.; Polini, M.; Novoselov, K. S. Graphene plasmonics. Nat. Photonics 2012, 6, 749-758. 
(28) Luo, X.; Qiu, T.; Lu, W.; Ni, Z. Plasmons in graphene: Recent progress and applications. Mat. Sci. Eng., $R$ 2013, 74, $351-376$.

(29) Nikitin, A. Y.; Guinea, F.; García-Vidal, F. J.; Martín-Moreno, L. Edge and waveguide terahertz surface plasmon modes in graphene microribbons. Phys. Rev. B 2011, 84, 161407.

(30) Chen, J.; Badioli, M.; Alonso-Gonzalez, P.; Thongrattanasiri, S.; Huth, F.; Osmond, J.; Spasenovic, M.; Centeno, A.; Pesquera, A.; Godignon, P.; Zurutuza Elorza, A.; Camara, N.; Javier García de Abajo, F.; Hillenbrand, R.; Koppens, F. H. L. Optical nano-imaging of gate-tunable graphene plasmons. Nature 2012, 487, 77-81.

(31) Lu, W. B.; Zhu, W.; Xu, H. J.; Ni, Z. H.; Dong, Z. G.; Cui, T. J. Flexible transformation plasmonics using graphene. Opt. Express 2013, 21, 10475-10482.

(32) Brar, V. W.; Jang, M. S.; Sherrott, M.; Lopez, J. J.; Atwater, H. A. Highly Confined Tunable Mid-Infrared Plasmonics in Graphene Nanoresonators. Nano Lett. 2013, 13, $2541-2547$.

(33) Chen, J.; Nesterov, M. L.; Nikitin, A. Y.; Thongrattanasiri, S.; Alonso-Gonz'alez, P.; Slipchenko, T. M.; Speck, F.; Ostler, M.; Seyller, T.; Crassee, I.; Koppens, F. H. L.; Mart'in-Moreno, L.; Garc'ia de Abajo, F. J.; Kuzmenko, A. B.; Hillenbrand, R. Strong Plasmon Reflection at Nanometer-Size Gaps in Monolayer Graphene on SiC. Nano Lett. 2013, 13, 6210-6215.

(34) Wang, B.; Zhang, X.; Yuan, X.; Teng, J. Optical coupling of surface plasmons between graphene sheets. Appl. Phys. Lett. 2012, 100.

(35) Christensen, J.; Manjavacas, A.; Thongrattanasiri, S.; Koppens, F. H. L.; García de Abajo, F. J. Graphene Plasmon Waveguiding and Hybridization in Individual and Paired Nanoribbons. ACS Nano 2012, 6, 431-440. 
(36) Francescato, Y.; Giannini, V.; Maier, S. A. Strongly confined gap plasmon modes in graphene sandwiches and graphene-on-silicon. New J. Phys. 2013, 15.

(37) Vasić, B.; Isić, G.; Gajić, R. Localized surface plasmon resonances in graphene ribbon arrays for sensing of dielectric environment at infrared frequencies. J. Appl. Phys. 2013, $113,1-7$.

(38) Zhao, Y.; Hu, X.; Chen, G.; Zhang, X.; Tan, Z.; Chen, J.; Ruoff, R. S.; Zhu, Y.; Lu, Y. Infrared biosensors based on graphene plasmonics: modeling. Phys. Chem. Chem. Phys. 2013, 15, 17118-17125.

(39) Jablan, M.; Buljan, H.; Soljačić, M. Plasmonics in graphene at infrared frequencies. Phys. Rev. B 2009, 80, 245435.

(40) Berini, P. Long-range surface plasmon polaritons. Adv. Opt. Photon. 2009, 1, 484-588.

(41) Wang, W.; Apell, P.; Kinaret, J. Edge plasmons in graphene nanostructures. Phys. Rev. $B 2011,84,085423$.

(42) Wang, W.; Apell, S. P.; Kinaret, J. M. Edge magnetoplasmons and the optical excitations in graphene disks. Phys. Rev. B 2012, 86, 125450.

(43) Rivas, J. G.; Vecchi, G.; Giannini, V. Surface plasmon polariton-mediated enhancement of the emission of dye molecules on metallic gratings. New J. Phys. 2008, 10, 105007.

(44) Gao, W.; Shu, J.; Qiu, C.; Xu, Q. Excitation of Plasmonic Waves in Graphene by Guided-Mode Resonances. ACS Nano 2012, 6, 7806-7813.

(45) Aydin, K.; Ferry, V. E.; Briggs, R. M.; Atwater, H. A. Broadband polarizationindependent resonant light absorption using ultrathin plasmonic super absorbers. Nat. Comm. 2011, 2. 
(46) Li, K.; Xiao, F.; Lu, F.; Alameh, K.; Xu, A. Unidirectional coupling of surface plasmons with ultra-broadband and wide-angle efficiency: potential applications in sensing. New J. Phys. 2013, 15, 113040.

(47) Vakil, A.; Engheta, N. Transformation Optics Using Graphene. Science 2011, 332, $1291-1294$. 


\section{Graphical TOC Entry}

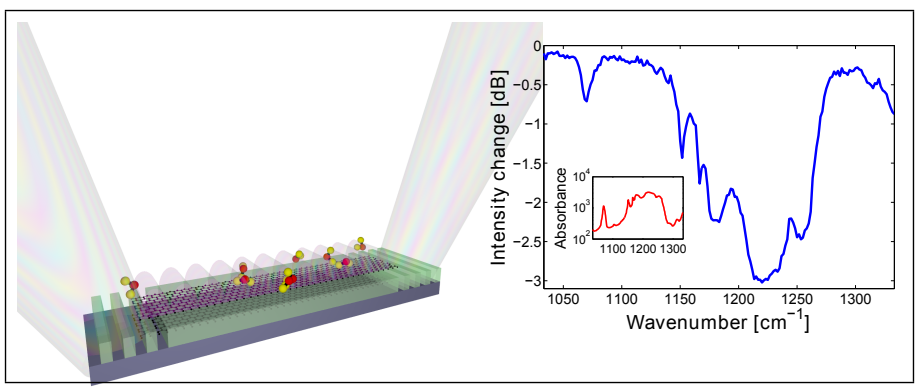

Graphene sandwiches can support broadband extremely confined surface plasmons which can detect highly diluted molecules with unprecedented sensitivity enabling an extended absorption spectrum to be measured. 